\title{
GEOMETRY AND MECHANICAL PROPERTIES OF A 3D-PRINTED TITANIUM MICROSTRUCTURE
}

\author{
Luboš Řehounek*, Petra HáJková, Petr VakrČka, Aleš Jíra
}

\author{
Czech Technical University in Prague, Faculty of Civil Engineering, Thákurova 7, 16629 Prague 6, Czech \\ Republic \\ * corresponding author: lubos.rehounek@fsv.cvut.cz
}

\begin{abstract}
Construction applications sometimes require use of a material other than construction steel or concrete - mainly in cases, where strength to weight ratio needs to be considered. A suitable solution to this problem are structures manufactured using the 3D printing process, as they have a very good strength to weight ratio (i.e.: Ti-6Al-4V $-\sigma_{\text {ult }}=900 \mathrm{MPa}$ and $\rho=4500 \mathrm{~kg} / \mathrm{m}^{3}$ ).

Trabecular structures are porous structures with local material characteristics identical to their commonly manufactured counterparts, but due to their geometry, they have different global mechanical properties and are suited for special applications. We designed and manufactured six variants of these structures and subjected them to uniaxial compression tests, nanoindentation tests and subsequently evaluated their differences and elastic moduli. The values of global moduli $\mathrm{E}$ are in the range of $2.55 \mathrm{GPa}-3.55 \mathrm{GPa}$ for all specimens.
\end{abstract}

KEYwORDs: Trabecular, porous, 3D printing, Young's modulus, nanoindentation.

\section{INTRODUCTION}

The trabecular structure functions as an alternative to conventional, homogeneous cross-sections of implants. Generally, it represents a 3D system of beams of certain length and composition embedded in a 3D matrix. In our case, they have an equal length and are represented by truss-like beams (Fig. 2).

Trabecular (porous) structures have many benefits as they can be used in applications where conventional structures are not sufficient. Among their many benefits is mainly their shapeability as the final shape only depends on the design. They can generally be used in applications where it is desirable to either provide a porous material due to the nature of the application or where reduced global modulus is needed [1]. Trabecular structures have, usually, lower moduli than conventional, homogeneous structures. This stems from the fact that they have a porous composition with a reduced cross-section (Fig. 2). They can also be used for the development of alternative geometrical solutions of stems of dental implants [2].

Trabecular structures are usually produced by $3 \mathrm{D}$ printing. Printing 3D metal structures is a process that falls under the category of additive manufacturing (AM). Additive manufacturing has many variants; our specific application used the technology of 3D printing of metals. This part is usually done using a computer-aided design (CAD) environment. Upon its completion, an STL model file is divided into thin cross sections [3, 4] and sent to the 3D printer to be processed. Up to this point, the process is similar to the common layer-by-layer 3D-printing of plastic.

What differentiates the process of printing metals from the standard technology is using a laser beam to melt down a layer of metal powder. During each cycle, the coater applies a thin layer of powder, which is processed by a laser at a pre-set melting point in a pre-determined order [4]. This process solidifies the loose powder into a 3D-layered object.

$3 \mathrm{D}$ printing is a very modern and perspective method in manufacturing of implants. It allows for very complex structures, which would not have been conceivable with traditional metalworking. It is also beneficial due to its ability to create small batches of products, thus being a more flexible way of manufacturing.

On the other hand, 3D printing still has its disadvantages, mainly heat dissipation problems and uneven material processing. From our own research, it is already known to us that uneven heat dissipation can cause cracks at the interface of the homogeneous and trabecular cross-sections. Dislocations of individual beams of the structures are also not uncommon and can cause wide impairments in the outcomes of the mechanical tests, rendering them effectively useless. These problems can, however, be solved by proper preparation of the $3 \mathrm{D}$ model, the printer assembly and by microscopic observation of the final structures.

\section{Materials And Methods}

\subsection{Description of Test Specimens}

The trabecular structures were manufactured by means of Selective Laser Melting (SLM) in an argon atmosphere of purity of 4.6. The material used for $3 \mathrm{D}$ printing was Ti-6Al-4V in the form of metal powder. The powder is named Rematitan (Concept Laser). After the 3D printing, the specimens were heat-treated to eliminate inhomogeneities which are created during the $3 \mathrm{D}$ printing process. The treatment 

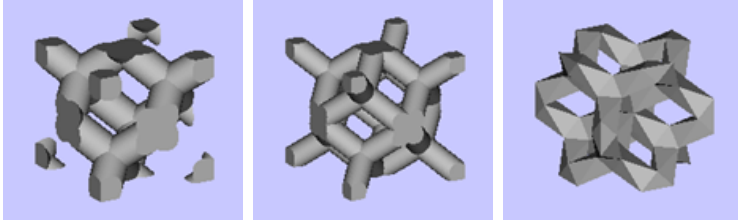

Figure 1. Basic element cells for creation of the test specimens.

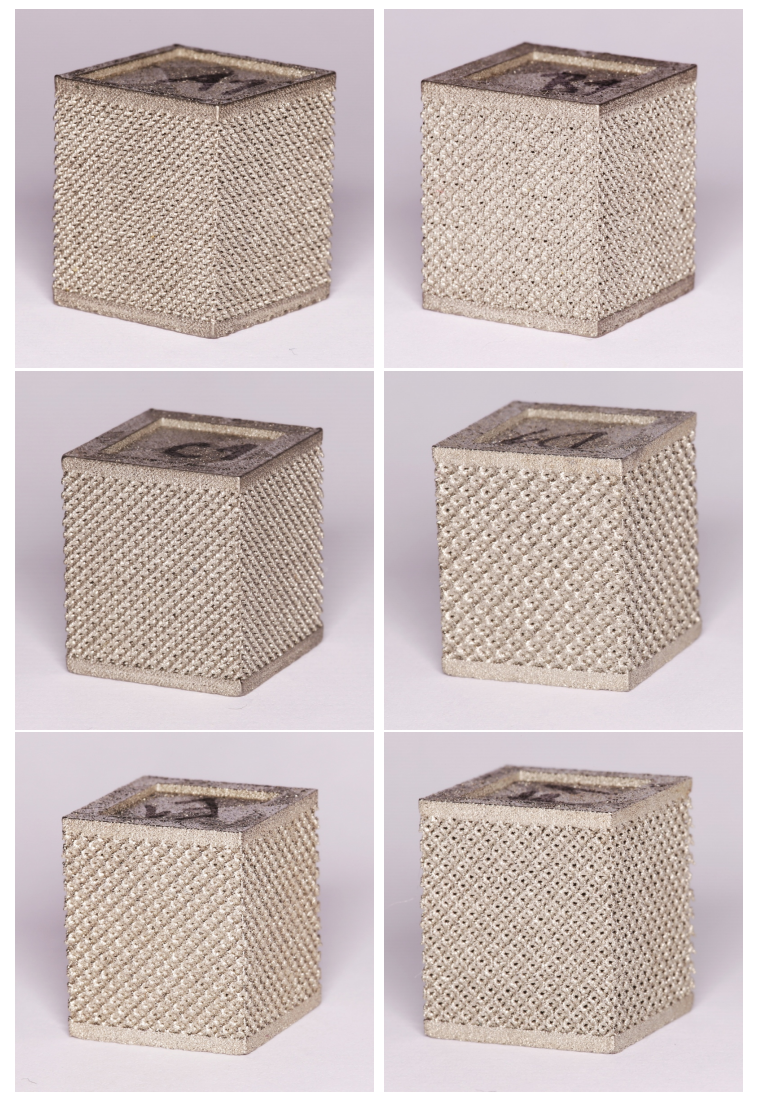

Figure 2. Real manufactured specimens A-F used for the uniaxial compression test.

was done in a vacuum furnace by gradual heating up to $840^{\circ} \mathrm{C}$ and subsequent quenching to $500^{\circ} \mathrm{C}$.

The geometrical solution of the trabecular structures was designed considering the optimal pore size for bone ingrowth. The desired pore size of 350-550 $\mathrm{\mu m}$ was achieved by using basic element cells labeled as Diamond, Dode Thick and Rhombic Dodecahedron (Fig. 1). Every basic element structure was manufactured in two variants of different density. A total od 21 test specimens was created. Every specimen comprises of a trabecular middle section (dimensions $14 \times 14 \times 14 \mathrm{~mm})$ and upper and lower homogeneous base (1 mm thick, Fig. 2).

The composition of the specimens is listed in Tab. 1 Specimens from the $\mathrm{A}$ and $\mathrm{C}$ series are designed using the Diamond element cell with density of 18 (A) and 14 (C) element cells per the width of the specimen. Specimens from the B and D series are designed using the Dode Thick elements with density of 14 (B) and 11.5 (D) element cells per the width of the specimen.

\begin{tabular}{ccc}
\hline Series & Structure type & Density \\
\hline A & Diamond & 18 \\
B & Dode Thick & 14 \\
C & Diamond & 14 \\
D & Dode Thick & 11.5 \\
E & Rhombic dodecahedron & 11.5 \\
F & Rhombic dodecahedron & 9.5 \\
\hline
\end{tabular}

TABLE 1. Structure type of individual specimens and their density (basic element count).
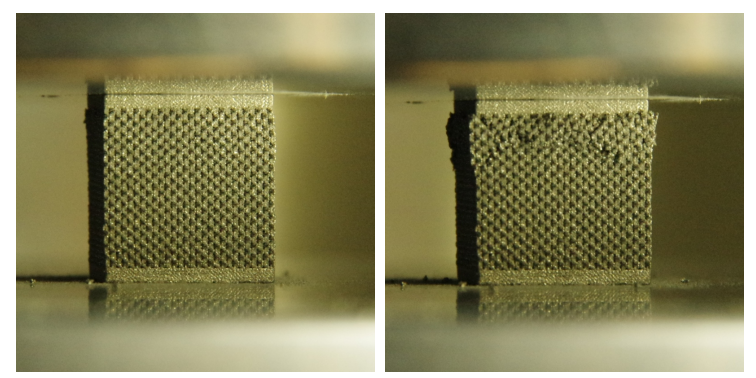

Figure 3. A specimen from the B series before loading (left) and during loading (right).

Element cells Rhombic Dodecahedron were used to design specimens from series $\mathrm{E}$ and $\mathrm{F}$ with density of $11.5(\mathrm{E})$ and $9.5(\mathrm{~F})$ per the width of the specimen.

\subsection{MACROMECHANICAL ANALYSIS}

Macromechanical analysis of the trabecular structures was carried out by a compression test. The compression test was performed in accordance with ISO 13314:2011 Compression test for porous and cellular metals [5]. The test was performed on the MTS Alliance RT-30 machine (CTU, Czech Republic) at room temperature. The loading direction for all structures was parallel to the building direction (perpendicular to the layers fabricated during 3D printing). Respecting the prescribed loading direction was important, as the trabecular structures are orthotropic due to character of $3 \mathrm{D}$ printing. The crosshead speed was set to $1.00 \mathrm{~mm} \cdot \mathrm{min}^{-1}$. The mechanical properties were determined from the stress-strain curves (Fig. 44.

Elastic modulus in compression $\mathrm{E}$ was calculated from the slope of the stress-strain curve in the linear elastic area in accordance with the ISO 13314 standard [5]. As the calculated elastic modulus represents the global parameter of the trabecular structure with pores, it has to differ (be lower) from the reduced modulus of the alloy itself. Evaluation of experimental data was done as described on Fig. 5 It is important to distinguish between the modulus of the material and modulus of the entire structure, as these two values vary dramatically.

For evaluation of Young's modulus E, we used the linear parts of the stress-strain curves of individual specimens obtained from the compression tests. Determination of the range of the loading curve is described 


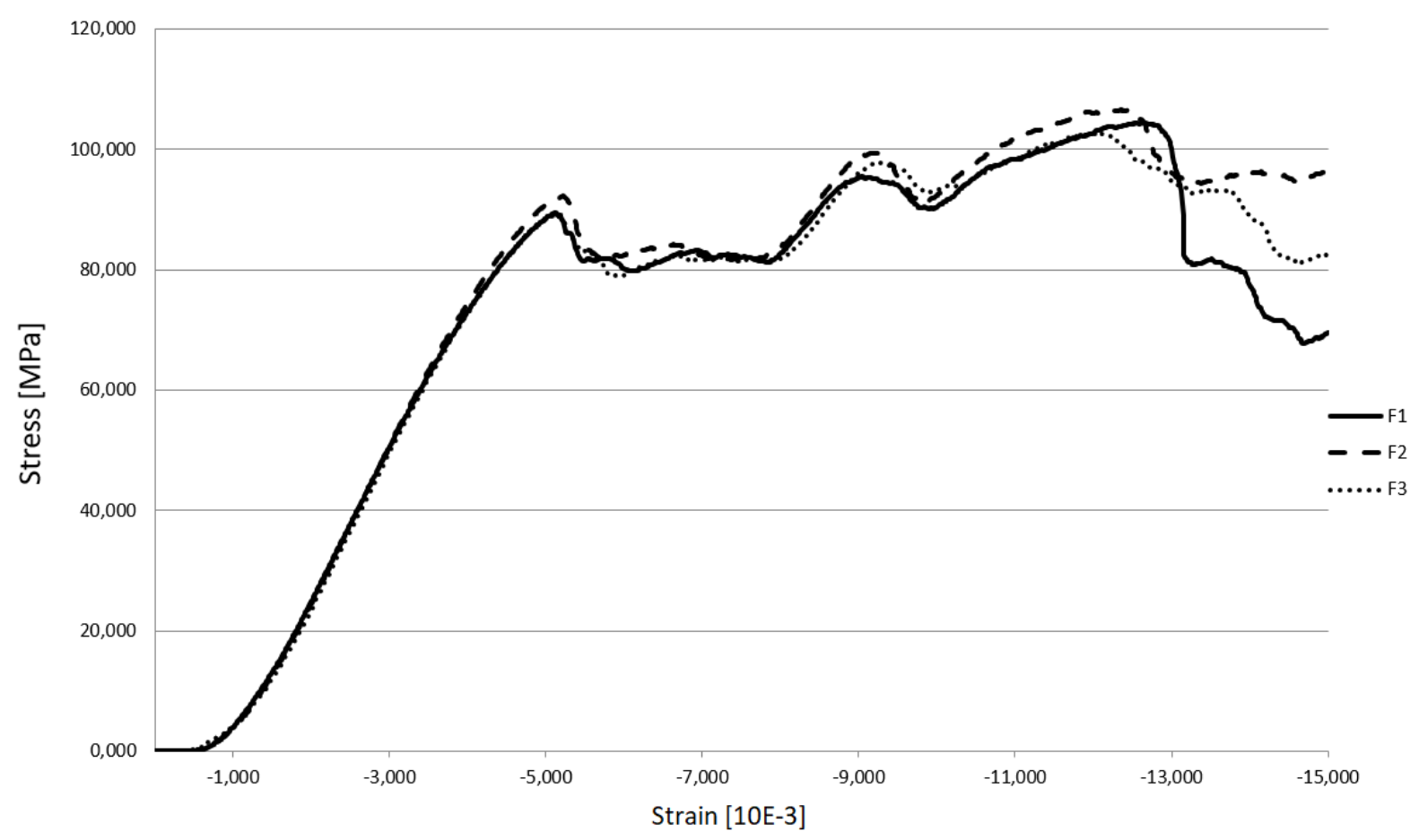

Figure 4. Stress-strain curves obtained from the compression test. Curves shown belong to the F specimen group. Note the method used to determine global E described in Fig. 5

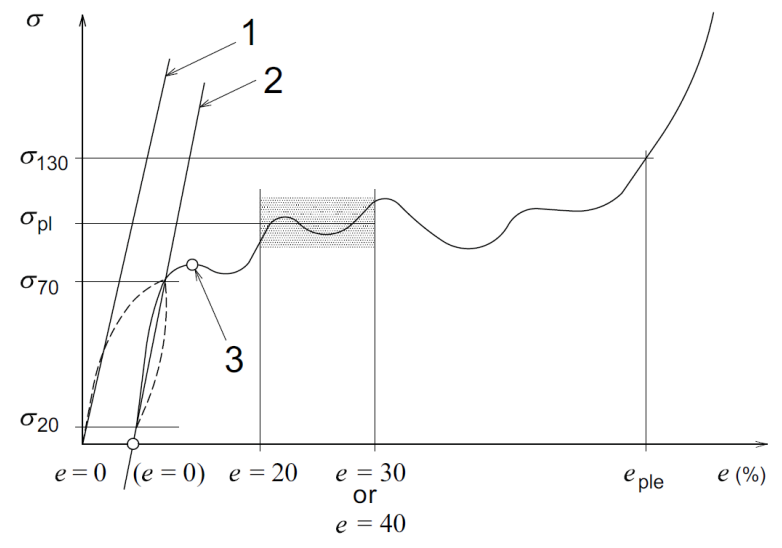

FIGURE 5. Stress-strain curve to determine the characteristic values from compression testing of porous and cellular metals 5]. Grayed out area in the graph represents the plateau stress, from which the mean value of $\sigma_{\mathrm{pl}}$ is calculated. The plateau stress is a mean value of stress calculated from stress values in between the range of strain $e=20 \%$ and $e=30 \% . \sigma_{70}$ and $\sigma_{20}$ then represent a range of values for caluculation of Young's modulus of the entire structure by the least squares method.

in [5] as well as Fig. 5] The final values of E for each specimen are listed in Tab. 2.

\subsection{MiCROMECHANICAL ANALYSIS}

The micromechanical analysis was performed on the CSM Instruments nanoindenter at the laboratory of CTU, Faculty of Civil Engineering, Prague. The specimens were embedded in epoxy resin, cut in transversal sections on a slow-rotating cutter and subsequently ground and polished to the desired level of quality with sandpapers of roughness of 1200-4000. To eliminate the surface tension and shear stiffness inside the atomic structure of the material, we used the method of directed force with cyclic loading. The loading force reached a value range of $10-70 \mathrm{mN}$ and was applied in 7 cycles with a loading/unloading speed of $180 \mathrm{mN} / \mathrm{min}$.

The micromechanical analysis proved the micromechanical properties dependent on the depth of the indent (magnitude of the loading force). From a contact depth of $\mathrm{h}_{\mathrm{c}} \quad 470 \mathrm{~nm}$ (corresponding loading force $30 \mathrm{mN}$ ), the trend of $\mathrm{E}_{\mathrm{r}}$ (reduced modulus) and $\mathrm{H}_{\mathrm{it}}$ (hardness) is constant. We can, therefore, assume the value of $30 \mathrm{mN}$ as a basic value of loading force. Values of micromechanical properties that correspond with this value are $\mathrm{E}_{\mathrm{r}}=118 \mathrm{GPa}$ and $\mathrm{H}_{\mathrm{it}}=5.19 \mathrm{GPa}$. For comparison, we also performed additional micromechanical tests on conventionally machined specimens, which turned out to be almost identical with values of $\mathrm{E}_{\mathrm{r}}=118 \mathrm{GPa}$ and $\mathrm{H}_{\mathrm{it}}=4.58 \mathrm{GPa}$. The tests on these specimens were carried out in the same way and environment as the tests of 3D-printed specimens.

\section{Results}

The results of the macromechanical compression tests are listed in Tab. 2 Series B, C and F were measured and evaluated successfully, but series A, D and E encountered problems during the compression test. The first 3 specimens from series A did not successfully 


\begin{tabular}{lcc}
\hline Series & Modulus of individual specimens $[\mathrm{GPa}]$ & Mean modulus of a series [GPa] \\
\hline A1 & Exceeded max. machine load & \\
A2 & Exceeded max. machine load & N/A \\
A3 & Exceeded max. machine load & \\
A4 & 2.69 & 2.78 \\
\hline B1 & 2.74 & \\
B2 & 2.99 & \\
B3 & 2.77 & \\
B4 & 2.63 & \\
\hline C1 & 3.47 & \\
C2 & 3.50 & N/A \\
C3 & 3.41 & \\
C4 & 3.48 & N/A \\
\hline D1 & Exceeded max. machine load & \\
D2 & Exceeded max. machine load & \\
D3 & Exceeded max. machine load & \\
\hline E1 & Exceeded max. machine load & \\
E2 & Exceeded max. machine load & \\
E3 & Exceeded max. machine load & \\
\hline F1 & 2.55 & \\
F2 & 2.62 & \\
F3 & &
\end{tabular}

TABLE 2. Values of all Young's moduli calculated from individual series of specimens according to ISO 13314 [5]. Some series of experiments exceeded the maximum value of appliable load, more on this topic in section Conclusions.

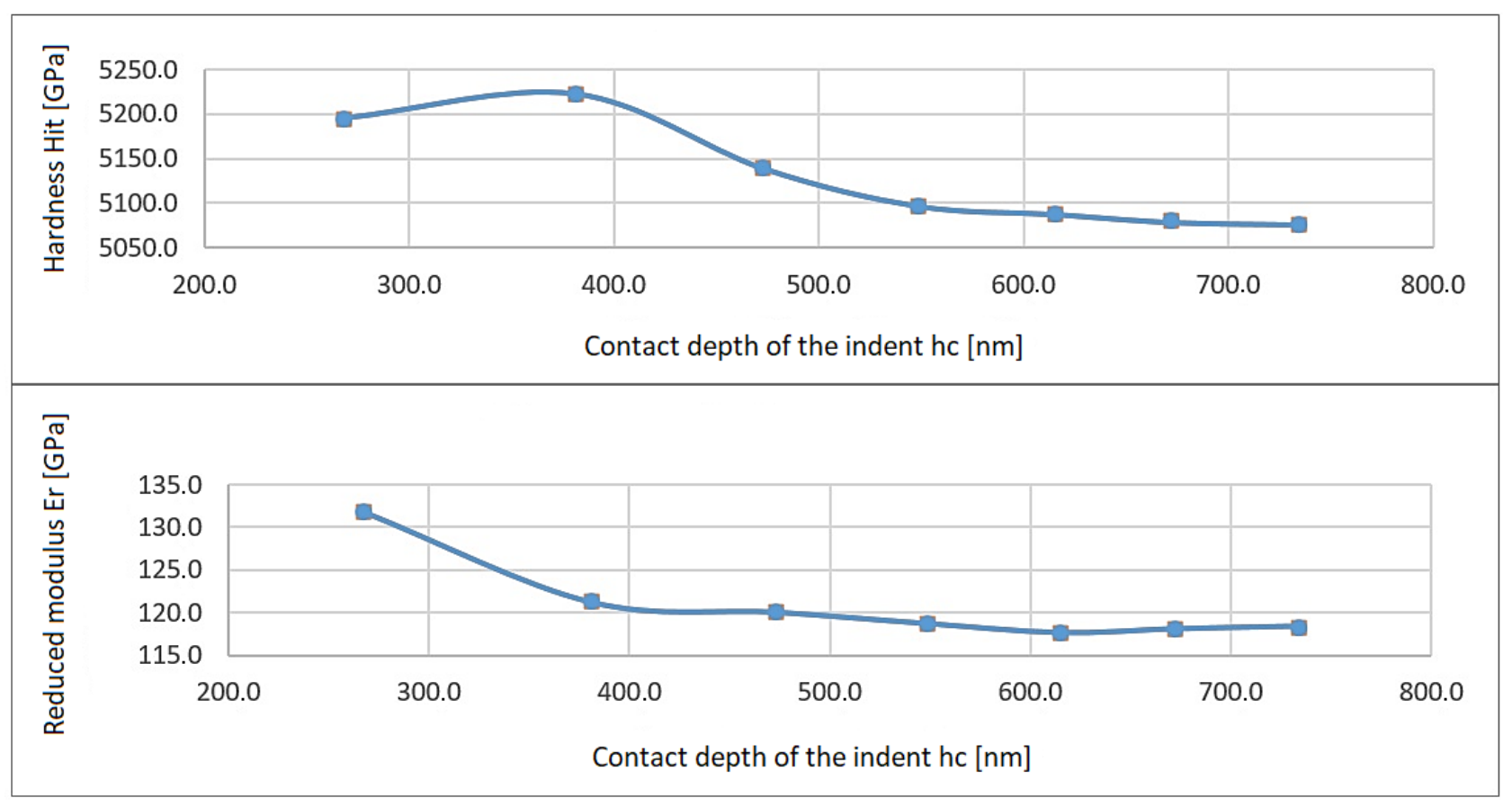

FIgURE 6. The trend of both measured micromechanical properties of porous specimens manufactured from a Ti-6Al-4V metal powder. The trend proved to be dependent on the depth of the indent.

reach a strain of 0.2 and therefore it was not possible to determine the values of moduli according to the used method [5] as it is required to calculate the plateau stress as a mean value of stress in the strain range of $0.2-0.3$. The problem arose as the machine prematurely reached its maximum value of load it can impose on the specimen. One specimen was successfully measured and is listed in Tab. 2 Series D and E 
encountered the same problem, but on a rather larger scale as the specimens were so stiff they did not even reach a strain of 0.1 before reaching the maximum machine load and ending the experiment prematurely.

\section{Conclusions}

Three out of a total 6 series of specimens failed the test on the MTS Alliance RT-30 machine as their geometrical solution provided a modulus so great it could not be measured. This shortcoming, although unfortunate, could not be accounted for beforehand as the geometrical solutions of the trabecular structures and their shape were developed with no prior expectations and knowledge of the total load needed to fully collapse the structures. The failure to measure the modulus stems from the fact that the experiment needs to be carried out at least to the range of 0.3 strain to meet the criteria of the used standard [5]. Three series of specimens were measured successfully and with good conformity amongst specimens of a given series (Tab. 2). The relation between their geometrical solution and values of moduli E (Fig. 2 and Tab. 22 can be taken into account in future development of a structure with specific requirements for its value of global modulus.

The micromechanical analysis also proved that 3Dprinted specimens have almost same mechanical properties on the micro level as specimens manufactured by conventional machining with hardness $\mathrm{H}_{\mathrm{it}}$ being slightly higher (12\% increase in 3D-printed specimens). This fact is probably caused by different heat treatment processes which are needed in case of 3Dprinted specimens.

\section{ACKNOWLEDGEMENTS}

The financial support provided by the Technology Agency of Czech Republic, project n.TJ01000328 and by the Faculty of Civil Engineering, CTU, Prague, project n.SGS17/168/OHK1/3T/11 is gratefully acknowledged.

\section{REFERENCES}

[1] T. Hasegawa, S. Kawabata, D. Takeda, et al. Survival of Brånemark System Mk III implants and analysis of risk factors associated with implant failure.

International journal of oral and maxillofacial surgery 46(2):267-273, 2016. DOI:10.1016/j.ijom.2016.10.014

[2] L. Řehounek, F. Denk, A. Jíra. Numerical Simulations of Static Strength of Novel Dental Implants. Acta Polytechnica 58(4):259, 2018. DOI:10.14311/AP.2018.58.0259.

[3] F. E. Wiria, J. Y. M. Shyan, P. N. Lim, et al. Printing of Titanium implant prototype. Materials and Design 31(SUPPL. 1):S101-S105, 2010.

DOI:10.1016/j.matdes.2009.12.050

[4] A. El-Hajje, E. C. Kolos, J. K. Wang, et al. Physical and mechanical characterisation of 3D-printed porous titanium for biomedical applications. Journal of Materials Science: Materials in Medicine 25(11):24712480, 2014. DOI:10.1007/s10856-014-5277-2

[5] I. Standard. ISO 13314 Mechanical testing of metals, ductility testing, compression test for porous and cellular metals. Reference number ISO

13314(13314):1-7, 2011. DOI:ISO 13314:2011 